\title{
Influencia de la reconstrucción del ligamento cruzado anterior sobre la calidad de vida, nivel de confianza y retorno al deporte: un seguimiento de 15 años
}

\author{
Influence of anterior cruciate ligament reconstruction on quality of life, level of \\ confidence and return to sports: a follow-up of 15 years \\ *Ana Paula Ramos Marinho; *Rodrigo Okubo
}

Ramos, A. \& Okubo, R. (2017). Influencia de la reconstrucción del ligamento cruzado anterior sobre la calidad de vida, nivel de confianza y retorno al deporte: un seguimiento de 15 años. Revista Ciencias de la Actividad Física UCM, $\mathrm{N}^{\circ} 18(2)$ julio-diciembre, 1-13.

\section{RESUMEN}

Antecedentes: la articulación de la rodilla es una de las más afectadas y lesionadas, siendo la lesión del ligamento cruzado anterior (ACL) la más frecuente entre ellas, alcanzando aproximadamente el 79\% de todas las lesiones de rodilla. Esta lesión, cuando involucra atletas, causa compromisos físicos, fisiológicos y especialmente psicológicos, considerando la alta tasa de incidencia o lesión contralateral después de regresar al deporte. Este compromiso a veces aleja al atleta del deporte, o disminuye su rendimiento. Toda esta limitación o deterioro afecta directamente la calidad de vida de los atletas. Caso clínico: en el presente estudio se realizó seguimiento de una atleta femenina de balonmano durante 15 años, que fue sometida a 3 reconstrucciones del LCA, para evaluar el deterioro físico, funcional y psicológico que estas cirugías causaron a lo largo de los años. Conclusión: se comprobó que el paciente se alejó por completo de las actividades posteriores a las cirugías, además de haber desarrollado un severo proceso de artrosis como consecuencia de un importante déficit muscular.

\section{PALABRAS CLAVE}

Ligamento cruzado anterior, atletas, balonmano, calidad de vida, retorno al deporte.

\begin{abstract}
Background information: the knee joint is one of the most affected and injured joints in the body, with the Anterior Cruciate Ligament (ACL) injury being the most common (about 79\%). However, when this injury involves athletes, not only does it cause physical damage, but physiological and, particularly, psychological as well, as it has a high recurrence rate or provokes a contralateral injury after returning to sports. This damage sometimes steers the athlete away from sports altogether, or performance decreases. Hence, these limitations or impairments directly affect the athletes' quality of life. Case report: in this study, monitoring or a follow up of a female handball athlete who had to undergo three reconstructions of the ACL was carried for 15 years in order to evaluate the physical, functional and psychological impairment that these surgeries caused over the years. Conclusion: it can be verified that the patient completely steered away from sports activities after the surgeries and developed a severe arthrosis process as a consequence of serious muscular deficit.
\end{abstract}

\section{Key words}

Anterior cruciate ligament, athletes, handball, quality of life, return to sports.

* Universidade do Estado de Santa Catarina, Brasil. 


\section{INTRODUCCIÓN}

Una de las principales lesiones que sufre la rodilla es la ruptura del ligamento cruzado anterior (LCA), con cerca del $79 \%$ de todas las lesiones en la articulación (Kraus et al., 2012; Rothenberg et al., 2016). La incidencia de esta lesión y de la cirugía para su reconstrucción se mueve en torno del 40/100.000 (Rothenberg et al., 2016), con practicantes de deporte a nivel competitivo que tienen un mayor riesgo de romper el LCA (Astur et al., 2016). La rehabilitación de la reconstrucción del LCA se relaciona directamente con el éxito de la cirugía. Una reconstrucción bien realizada del LCA necesita de una completa rehabilitación física en miras de permitir que el paciente retome de la mejor manera posible sus actividades de la vida diaria y deportiva (Astur et al., 2016).

El proceso postoperatorio generalmente es caracterizado por síntomas como dolor y pérdida del control neuromuscular, principalmente del cuádriceps, lo que provoca malestar e impide que el paciente pueda realizar actividades simples como bañarse y moverse con autonomía. La recuperación del control muscular y el alivio de los síntomas dolorosos es fundamental para que el paciente pueda pasar por un complejo proceso de recuperación funcional (Trees et al. 2011). Todo ese largo proceso que puede tener una duración de 6 meses tiene implicancia en la calidad de vida de esos pacientes y genera dependencia para algunas tareas (Garratt et al., 2008).

El acompañamiento clínico y funcional a largo plazo después de la reconstrucción del LCA, asociado a su influencia en la calidad de vida de esos pacientes no está muy investigada en Brasil.

Un estudio realizado por Gifctad et al. (2013), demostró que no había diferencia significativa entre la pierna operada y la sana entre la inestabilidad de la rodilla, nivel de actividad o la condición funcional del mismo, medida de manera subjetiva y la calidad de vida. Sin embargo, Wipfler et al. (2013) indica una diferencia significativa a favor de la pierna operada en relación a la inestabilidad y test funcionales.
El paciente que pasa por una cirugía de reconstrucción del LCA, sufre algunas alteraciones psicológicas por miedo de una nueva lesión, lo que acaba comprometiendo la calidad de vida del individuo, ya sea por la inestabilidad persistente de la rodilla, dolor o cualquier otro síntoma que le impida o dificulte retomar sus actividades deportivas (Heijne, Hagstromer \& Werner, 2015).

El objetivo de este trabajo es realizar una descripción de un estudio de acompañamiento $($ follow-up) de 15 años de una paciente que fue sometida a la reconstrucción del LCA bilateral, con el fin de verificar el compromiso físico, funcional, psicológico y la influencia que el proceso de reconstrucción tuvo en su calidad de vida. En virtud de que se trata de un follow-up largo y la reconstrucción haya sido bilateral, se espera que los cambios sean más evidentes e importantes en todos los aspectos

\section{METODOLOGÍA}

\section{Descripción del caso}

Muestra: paciente de sexo femenino de 14 años (1.65 m y $55 \mathrm{~kg}$ ), físicamente activa, estudiante de enseñanza media y atleta de hándbol. La paciente realizaba entrenamiento muscular 2 a 3 veces por semana y entrenamiento técnico y táctico específico de la modalidad, 6 veces por semana. La primera lesión se produjo durante los juegos interescolares en el año 2000, donde sufrió una torsión en la rodilla derecha. La paciente relató que sintió un chasquido seguido de un dolor muy intenso que le impidió incluso caminar, quien fue inmediatamente remitida a un ortopedista, quien verificó la positividad de los test de Lachman, cajón anterior y pivot-shift. El diagnóstico fue complementado con un examen de resonancia magnética, que demostró discontinuidad de las fibras del LCA, específicamente de las fibras en la región intercondilar, confirmando entonces la lesión completa del LCA, además de haber recibido el diagnóstico de la lesión en el cuerpo y el cuerno posterior del menisco medial asociado. En virtud de la edad precoz y de las atribuciones escolares, la primera cirugía se realizó un año después de la lesión. La cirugía fue realizada 
en la rodilla derecha cuando la paciente tenía 15 años de edad, el año 2001.

El procedimiento realizado fue la reconstrucción utilizando el tercio medio del tendón patelar con injerto además de una meniscectomía parcial del menisco medial, siendo retirado el cuerpo y el cuerno posterior del mismo. No hubo complicaciones postoperatorias y la rehabilitación fue realizada de acuerdo al protocolo acelerado de Shelbourne (Shelbourne \& Gray, 1997). Después de cinco meses de rehabilitación y refuerzo muscular, la paciente fue autorizada para retomar las actividades deportivas.

El año 2006, la paciente sufrió una nueva torsión, pero en la rodilla izquierda, durante un partido de hándbol en los juegos internos de la universidad. Relató que durante un movimiento de detención brusca y cambio de dirección sufrió un empujón y una torsión debido al desequilibrio. Indicó además que el dolor fue muy intenso lo que le impidió mantenerse en el campo de juego, siendo llevada inmediatamente al hospital, donde fue evaluada y sometida a una resonancia magnética en la que se evidenció una ruptura completa de las fibras del LCA y edema en las fibras del ligamento colateral medial, además de una lesión en el menisco medial. Nuevamente fue sometida a una reconstrucción en cuanto de comprueba el diagnóstico; en esta oportunidad se utilizan como injerto, tendones flexores (semitendinoso y semimembranoso). El procedimiento quirúrgico no tuvo complicaciones y para el proceso de rehabilitación se utilizó el protocolo acelerado de Noyes sin que haya ocurrido interrupción en el periodo (Heckmann, Noyes \& Barber-Westin, 2009).

Después de cinco meses de rehabilitación la paciente comenzó a sentir dolores en la región medial de la rodilla derecha. Después de la evaluación clínica del médico, fue diagnosticada una insuficiencia del ligamento cruzado de la rodilla derecha, intervenida el año 2001; fue operado nuevamente, además de hacer una revisión de la reconstrucción del LCA, utilizándose en esa oportunidad los tendones flexores como injerto. Durante el procedimiento quirúrgico, se observó la presencia de un cuadro severo de degeneración del cartílago articular, principalmente en el cóndilo medial de la rodilla derecha, probablemente relacionado a la insuficiencia de estabilidad y la ausencia del menisco en esa región, como resultado de la meniscectomía realizada el 2001. No hubo interrupciones en el procedimiento quirúrgico y en la rehabilitación, que fue realizada utilizando el protocolo para la revisión del LCA de Hoyes (Heckmann, Noyes \& Barber-Westin, 2009).

Después de 8 meses de rehabilitación, la paciente recibió el alta médica y la fisioterapia, siendo autorizada a retomar sus actividades. Sin embargo, esa autorización no fue completa, la paciente recibió orientaciones en relación al tipo de actividad que debería realizar y evitar, con el propósito de impedir un empeoramiento del cuadro degenerativo y al mismo tiempo impedir que haya un riesgo de una nueva lesión ligamental. Con esas recomendaciones, la paciente fue autorizada para realizar actividades físicas sin impacto, giros y pivot, en consecuencia fue orientada a abandonar el hándbol. Actividades como corridas fueron autorizadas desde que la paciente alcanzara los niveles esperados de fuerza muscular y sentirse segura para tal actividad.

\section{Instrumentos y Follow-up}

La paciente fue evaluada periódicamente, se utilizó el cuestionario de Lysholm (Pecccin, Ciconelli \& Cohen 2006), la escala modificada de Cincinnati (Bentley et al. 2003) y la escala de evaluación de la actividad física de Tegner (Tegner \& Lysholm, 1985), después de 2, 4 y 6 meses de cada cirugía.

Fueron realizadas evaluaciones isocinéticas con el objetivo de verificar la condición de fuerza de la musculatura del cuádriceps e isquiotibiales. Para esos efectos se utilizó un dinamómetro de la marca Cybex-CSMi, modelo HumacNorm, 2009, con una tasa de adquisición de la señal en $500 \mathrm{~Hz}$. La evaluación isocinética fue realizada en la paciente después de la revisión del LAC de la rodilla derecha en el 2008, y repetida en el 2012 y 2016. 
El protocolo de la evaluación utilizada se basó en las recomendaciones de la APTA-Asociación Americana de Fisioterapia (Logerstedt et al., 2010), además de los modelos utilizados en otros estudios similares (Almosnino et al., 2014, Bryant, Pua, \& Clark, 2009).

El profesional que realizó los test, fue un fisioterapeuta con cinco años de experiencia en biomecánica. La calibración del equipamiento fue realizada de acuerdo a las orientaciones en el manual del fabricante.

A la paciente se le presentó el equipamiento e informó como realizar el test, su finalidad, además de aclarar los posibles síntomas que pudiera experimentar durante y después de la realización del examen. Al aplicar el test se adoptó la posición exigida para la evaluación de la rodilla en que la paciente se ubicó sentada, con la columna lumbar totalmente apoyada y la cadera flexionada en $85^{\circ}$. El respaldo de la silla fue ajustado para la región poplítea de la evaluada, considerando distanciarla del borde del asiento de la silla en una pulgada, no limitando así el movimiento de flexión completa. La paciente fue estabilizada en la cadera a través de un cinturón en la región del tronco y la cintura, y la pierna contra lateral fijada por una banda de velcro. El eje de la rotación de la rodilla evaluada fue alineada con el eje del dinamómetro, y el brazo de la palanca ajustado al tamaño de la pierna, siendo ajustada a una pulgada por encima del maléolo lateral, permitiendo el libre movimiento del tobillo durante la prueba.

El protocolo consideró 5 repeticiones submáximas para familiarizarse con el equipamiento. Las repeticiones fueron realizadas a $60 \%$, con una amplitud del movimiento de $100^{\circ}$. Después de las repeticiones de familiarización, se aplicó el test consistente en la ejecución de 3 repeticiones máximas a la misma velocidad y amplitud que en el caso de la familiarización. Durante el test máximo, la paciente recibió incentivo verbal por parte del fisioterapeuta evaluador, que monitoreaba la intensidad del dolor durante el test, impidiéndole continuar si el dolor fuese de un valor mayor a 0.5 en la escala analógica del dolor.
Todo el procedimiento fue realizado de manera bilateral, iniciando con la pierna sana y en el caso de la evaluación realizada tras la revisión del LCA de la rodilla derecha, se inició con la rodilla izquierda.

El valor registrado en el examen fue siempre el mayor de las tres repeticiones. El test fue realizado de manera concéntrica y excéntrica, a la misma velocidad y amplitud, siempre respetando la tolerancia de la paciente.

Con el fin de verificar cuanto habían alterado estos procedimientos, la calidad de vida de la paciente, en el 2008, fue aplicado el instrumento ACL deficiency quality of life (ACLQOL) (Mohtadi, 1998).

Los puntajes de la escala ACL-QOL, van de 0 a 100, y la paciente puntéa el nivel de veracidad o la importancia de la información en una media de las 32 preguntas. Además de eso, se aplicó el instrumento ACL-Return to Sport after Injury (ACL-RSI), con el propósito de verificar la condición psicológica de la paciente para retornar a la práctica del deporte, que puntea de 0 a 10 en 12 preguntas y una media de los valores, donde la media de 10, permite afirmar que el individuo está totalmente confiado en la capacidad de su rodilla para la práctica deportiva y la media 0 , totalmente insegura (Webster, Feller \& Lambros, 2008).

En el 2012 y en el 2016, se hicieron nuevas evaluaciones con la aplicación de los cuestionarios junto a la evaluación isocinética.

\section{RESULTADOS}

Actualmente la paciente tiene 30 años con un hijo de 3 años y no practica ningún tipo de actividad. No presenta dolores en las rodillas, pero comenta que siente dificultades para realizar algunas actividades, en especial al golpear un objeto en el suelo, y comenta tener mucha dificultad al levantarse, además de la crepitación permanente de las rodillas. Además, la paciente relata que no es capaz de mantener en brazos a su hijo por mucho tiempo, pues siente sus piernas muy cansadas. En relación al peso corporal, se mantiene el mismo que tenía an- 
tes, por lo tanto no es esta variable la que influya o esté relacionado con el dolor y malestar. Los valores de los instrumentos funcionales se presentan en la Tabla 1.

Sobre la evaluación funcional subjetiva, evaluada a través de la escala modificada de Cincinnati, la paciente tuvo el menor resultado después de dos meses del preoperatorio luego de la reconstrucción de la rodilla derecha con apenas 24 puntos, clasificándola como muy mala. Las medidas a los seis meses, 2008, 2012 y 2016, fueron prácticamente equivalentes, clasificada como excelente para las dos rodillas conforme la Tabla 2 (Bentley et al. 2003).

En relación a la evaluación realizada por medio de la escala de Lysholm, el menor valor fue a los dos meses después de la reconstrucción de la rodilla derecha, clasificada como muy mala, y el mejor valor en el 2008 de la rodilla izquierda, cuyo resultado fue el máximo valor. La evolución de las mediciones se observan en la Tabla 1 (Peccin, Ciconelli \& Cohen, 2006)

Los resultados de las evaluaciones de la escala de Tegner, muestran que la paciente no consiguió retomar el nivel deportivo que tenía antes de las cirugías. Los puntajes fueron de cero, caracterizada por ninguna o muy poca actividad realizada por problemas en las rodillas, en el postoperatorio de dos meses en las tres evaluaciones. En las de cuatro meses se clasificó en puntaje uno (1) caracterizada como actividad muy leve (sedentaria). En las evaluaciones de seis (6) meses quedó clasificada en el puntaje 6 , considerada una actividad deportiva recreacional, por lo menos cinco veces por semana. En la evaluación realizada el 2008, el puntaje fue de 5, caracterizada como una actividad deportiva recreacional de 2 veces por semana. En el 2012, el puntaje fue de 4 , caracterizada por una actividad laboral de moderada intensidad y en el 2016 el puntaje fue de 3, que se caracteriza por una actividad leve (Tabla 3) (Tegner \& Lysholm, 1985).
La medición sobre calidad de vida fue aplicada en el 2008, 2012 y 2016. En las tres evaluaciones respecto a la influencia de las cirugías de la rodilla izquierda, como para la de la rodilla derecha, los valores sumados fueron muy bajos respecto del ideal, demostrando una gran alteración en la calidad de vida de la paciente (Tabla2 y Figura 1). Los valores de la rodilla izquierda estuvieron más altos que los de la rodilla derecha en casi todas las situaciones, evidenciadas especialmente en las del 2016. La pregunta 21: "Do you have to concern yourself with general safety issues (i.e., carrying small children, working in the yard) with respect to your ACL-deficient knee?", tuvo una puntuación máxima en las evaluaciones del 2008, 2012 y 2016 en ambas rodillas. Y en la pregunta 31: "How fearful are you of reinjuring your knee?”, tuvo puntuaciones mínimas en las tres mediciones de las rodillas. La media de los valores para los tres años fueron $51,61,59$ y 51,64 y 69 para la rodilla derecha e izquierda respectivamente. Se advierte que la media de los valores de la rodilla izquierda fueron ligeramente mayores que la de la rodilla derecha, demostrando una mayor influencia en la calidad de vida de la paciente y mayores preocupaciones con la rodilla derecha (Mohtadi, 1998).

La medición psicológica, que buscaba evaluar la seguridad de la paciente para retomar las actividades físicas, fue realizada por medio del cuestionario ACL-RSI, en el que los valores medios de la paciente 5.4, 4.6 y 4.8 para los años 2008, 2012 y 2016 respectivamente, demostraron la alta inseguridad sentida por la paciente en relación a las rodillas. No hubo sumas máximas o mínimas en ninguna de las evaluaciones (Webster, Feller \& Lambros, 2008).

En relación a las evaluaciones isocinéticas, fueron realizadas 3 mediciones concéntricas y excéntricas de forma bilateralmente en los tres años (Tabla 3). La evolución de las fuerzas musculares puede ser observada en las Figuras 2 y 3. 
Tabla 1

Valores de la escala modificada de Cincinatti, escala de Lysholm y escala de Tegner.

\begin{tabular}{lcccccc}
\cline { 2 - 7 } & \multicolumn{6}{c}{ Períodos de evaluación } \\
\cline { 2 - 7 } & 2 meses & 4 meses & 6 meses & 2008 & 2012 & 2016 \\
\hline Cincinnati & 52 & 82 & 90 & 88 & 90 & 87 \\
\hline 1a Cirugía - Rodilla Derecha & 24 & 52 & 84 & 88 & 90 & 87 \\
\hline 2a Cirugía - Rodilla Derecha & 42 & 81 & 92 & 92 & 91 & 91 \\
\hline Joelho Esquerdo & & & & & & \\
\hline \multicolumn{1}{c}{ Lysholm } & 36 & 75 & 95 & 94 & 99 & 94 \\
\hline 1a Cirugía - Rodilla Derecha & 28 & 59 & 89 & 94 & 99 & 94 \\
\hline 2a Cirugía - Rodilla Derecha & 44 & 67 & 94 & 100 & 99 & 99 \\
\hline Rodilla Izquierda & & & & & & \\
\hline Tegner & 0 & 1 & 6 & 5 & 4 & 3 \\
\hline 1a Cirugía - Rodilla Derecha & 0 & 1 & 6 & 5 & 4 & 3 \\
\hline 2a Cirugía - Rodilla Derecha & 0 & 1 & 6 & 5 & 4 & 3 \\
\hline Rodilla Izquierda & & & & & & \\
\hline
\end{tabular}

Fuente: propia del autor.

\section{Tabla 2}

Valores de los cuestionarios ACL-QOL y ACL-RSI.

\begin{tabular}{ccccc}
\hline & & \multicolumn{3}{c}{ Períodos de evaluación } \\
\cline { 3 - 5 } & & 2008 & 2012 & 2016 \\
\multirow{3}{*}{ ACL-QOL } & Direito & 51 & 61 & 59 \\
& Esquerdo & 51 & 64 & 69 \\
\hline \multirow{3}{*}{ ACL-RSI } & 2008 & 2012 & 2016 \\
& & 5,4 & 4,6 & 4,8 \\
\hline
\end{tabular}

Fuente: propia del autor.

\section{Tabla 3}

Valores medios de fuerza muscular, en Newtons, de la musculatura extensora y flexora de rodilla derecha e izquierda, en los períodos de evaluación del 2008, 2012 e 2016.

\begin{tabular}{cccc}
\hline & \multicolumn{3}{c}{ Períodos de avaliação } \\
\cline { 2 - 4 } & 2008 & 2012 & 2016 \\
Concêntrico Direito & $135,0 \mathrm{~N}$ & $119,0 \mathrm{~N}$ & $90,0 \mathrm{~N}$ \\
Concêntrico Esquerdo & $141,0 \mathrm{~N}$ & $118,0 \mathrm{~N}$ & $98,0 \mathrm{~N}$ \\
Excêntrico Direito & $160,0 \mathrm{~N}$ & $135,0 \mathrm{~N}$ & $115,0 \mathrm{~N}$ \\
Excêntrico Esquerdo & $165,0 \mathrm{~N}$ & $137,0 \mathrm{~N}$ & $126,0 \mathrm{~N}$ \\
\hline Concêntrico Direito & $80,0 \mathrm{~N}$ & $72,0 \mathrm{~N}$ & $63,0 \mathrm{~N}$ \\
Concêntrico Esquerdo & $88,0 \mathrm{~N}$ & $75,0 \mathrm{~N}$ & $68,0 \mathrm{~N}$ \\
Excêntrico Direito & $100,0 \mathrm{~N}$ & $93,0 \mathrm{~N}$ & $65,0 \mathrm{~N}$ \\
Excêntrico Esquerdo & $102,0 \mathrm{~N}$ & $90,0 \mathrm{~N}$ & $78,0 \mathrm{~N}$ \\
\hline
\end{tabular}

Fuente: propia del autor. 


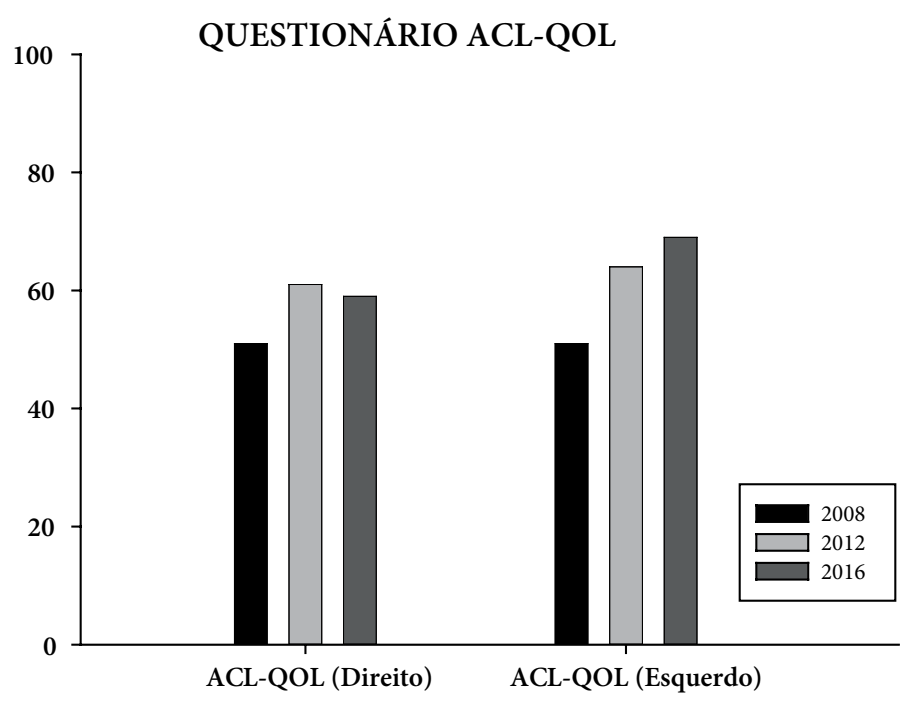

Figura 1. Gráfico evolutivo de los escores de la evaluación ACL-QOL. Fuente: propia del autor.

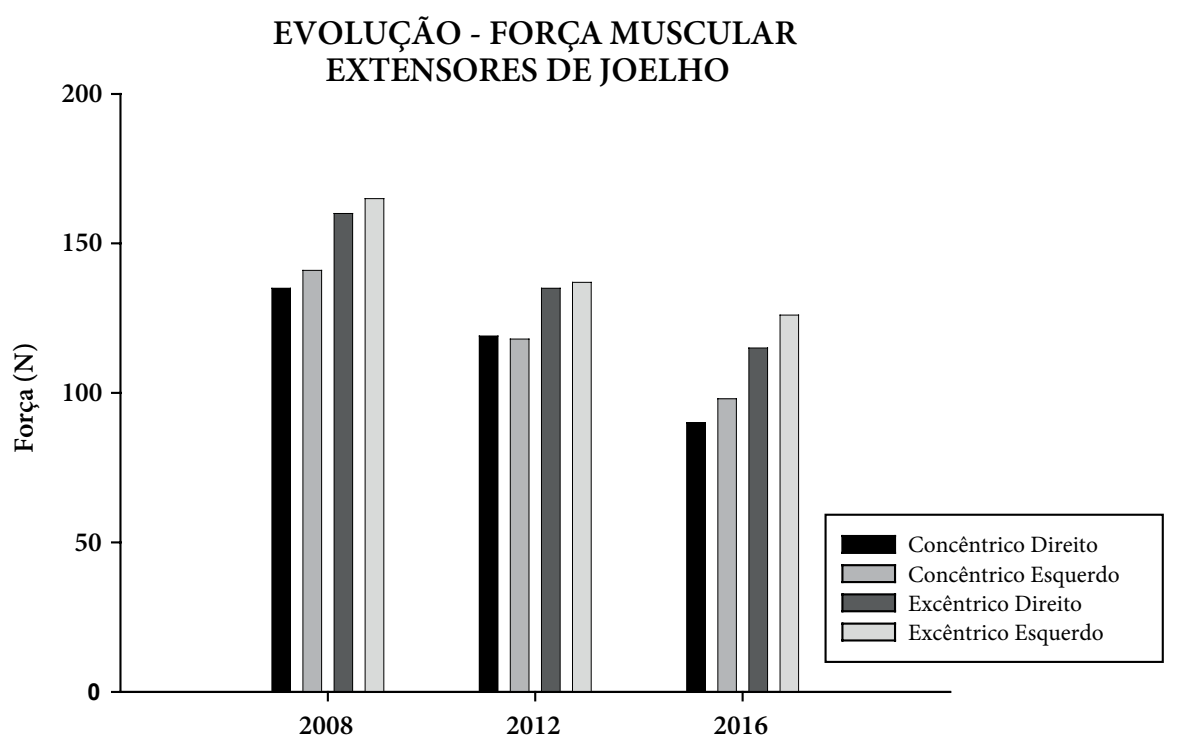

Figura 2. Gráfico evolutivo de las fuerzas musculares extensoras de rodilla derecha e izquierda, en los períodos de evaluación del 2008, 2012 e 2016. Fuente: propia del autor. 


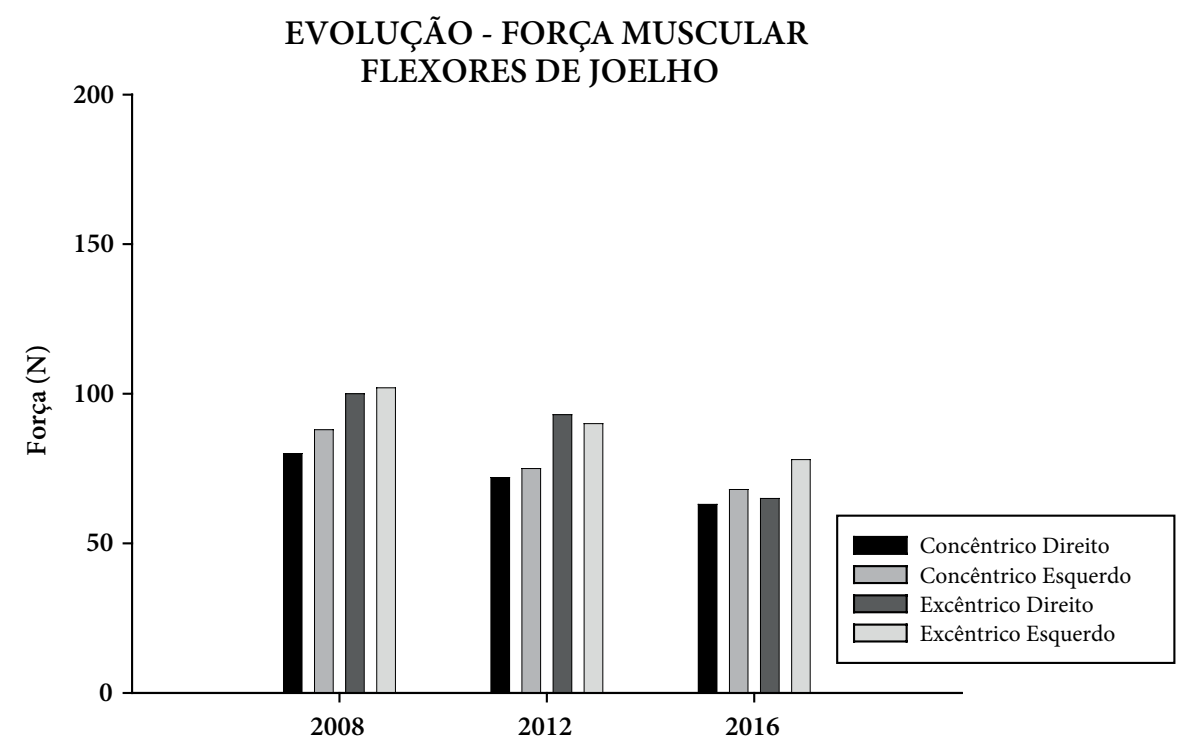

Figura 3. Gráfico evolutivo de las fuerzas musculares flexores de rodilla derecha e izquierda, en los períodos de evaluación del 2008, 2012 e 2016. Fuente: propia del autor.

\section{DISCUSIÓN}

El presente relato de caso describe los datos de una ex atleta de sexo femenino, que sufrió una lesión completa del LCA bilateralmente y fue sometida a procedimiento de reconstrucción de los mismos. La historia clínica de la paciente corrobora los datos de un estudio realizado el 2011 que apuntó a un índice de lesión contra lateral después de una lesión completa del LCA de más del 15\% (Wright et al. 2011). Swärd, en la revisión publicada en el 2010 (Swärd, Kostogiannis \& Roos, 2010), afirma que explicar los verdaderos motivos de la lesión contra lateral del LCA son muy complicados, pues existen innumerables factores asociados. Ellos afirman que existen factores extrínsecos (individuales) e intrínsecos (ambientales) involucrados en la lesión. Entre los intrínsecos, el factor más concordante es el sexo, donde las mujeres presentan un aumento en el riesgo debido por la disminución de la fosa intercondilar y del tamaño del LCA, y el factor extrínseco más común es la calidad de la zapatilla utilizada con la tracción que tiene en la superficie del juego. Considerando los factores intrínsecos y extrínsecos más comunes, se percibe que la paciente se encuadraba en los dos, por ser de sexo femenino y practicar un deporte con muchos movimientos de giros y pivotes, que exige una fuerte tracción y frición entre el suelo y el pie, aumentando la fuerza de torción de los movimientos.

Otro factor fuertemente involucrado con la incidencia de la lesión del LCA, es el equilibrio muscular entre el cuádriceps e isquiotibiales, que algunos autores consideran como un factor intrínseco, por ser una cualidad individual de la característica de las fibras y otros extrínsecos por tener una característica específica para el deporte practicado, posición y tiempo de entrenamiento (Xaverova et al., 2015). Los valores de referencia poblacional adoptados para evaluar la condición de fuerza de la paciente se basaron en el estudio de Danneskiold-Samsoe et al. (2009), que determina los valores de torque concéntricos en $132 \mathrm{~N}$ para extensores y $74.4 \mathrm{~N}$ para flexores y valores cerca del $30 \%$ mayores de torque excéntrico (Xaverova et al., 2015). En relación con los valores poblacionales y los de la paciente, se percibe respecto a los valores concéntricos, que presentaba, con excepción de la evaluación del 2008, valores inferiores a los predichos para su franja etaria, de acuerdo a 
la referencia citada. Las evaluaciones del 2012 y 2016 detectaron un déficit del $9.8 \%$ y $31.8 \%$ de los extensores derechos y el $10.6 \%$ y $25.8 \%$ de los extensores izquierdos. En relación a los flexores, los déficit registrados fueron del 3.2\% en el derecho en el 2012 y del $15.3 \%$ y $8.6 \%$ en la pierna derecha e izquierda respectivamente en el año 2016. Al analizar los datos excéntricos predichos, se percibe que la paciente queda por debajo de los valores de normalidad en todas las evaluaciones para las dos musculaturas medidas, siendo su mayor déficit del $20.6 \%$ referente a la evaluación excéntrica del flexor de la pierna derecha realizada el 2016, y el menor de $0.6 \%$ referente a la evaluación de las misma pierna, realizada en el 2012.

Estudios específicos en atletas de hándbol, afirman que existe una relación ideal entre torque del isquiotibiales y cuádriceps que debe estar entre 0.65 y 0.71 . Esa relación se da mucho en función de las características de la modalidad que considera valencias de explosión, fuerza, agilidad, velocidad, cambios de dirección, además de ser un deporte de mucho contacto (Xaverova et al., 2105).

Se percibe que la referencia utilizada con valores poblacionales, no se condice con las sugerencias específicas de la modalidad, debido a que pueden haber colaborado los residuos de la lesión, considerando que la paciente presentaba en todas las evaluaciones valores por debajo de los utilizados para la referencia poblacional, y con el equilibrio inadecuado entre las musculaturas para la modalidad.

Además, los factores intrínsecos, relacionados con la calidad de vida y la condición psicológica del atleta son muy importantes. Esos factores están íntimamente conectados, pues cuestiones como el miedo de recaída e inseguridad durante el gesto deportivo pueden contribuir a la disminución de la calidad de vida del atleta, derivando, como situaciones extremas, el abandono de la actividad con valores de hasta el 50\% del retiro (Werbster, Feller \& Lambros, 2008, Filbay et al., 2014, Johnsson et al., 2016). Esos factores fueron indicados por la paciente, siendo el principal o el único motivo del abandono de las actividades deportivas; la inseguridad y el miedo de una nueva lesión, además de los largos periodos de rehabilitación y las expectativas para un nuevo retorno frustrado al deporte. Factores como la lesión meniscal asociada, lesión del complejo póstero lateral y la presencia de osteoartritis instalada son los principales factores identificados como responsables por la baja calidad de vida relatada por los individuos sometidos a la reconstrucción del LCA (Filbay et al., 2015).

Además del miedo y la inseguridad, la paciente tenía compromiso articular en su rodilla. Con el fin de minimizar los efectos del deterioro de la meniscectomía y de la lesión del cartílago existente, hizo uso de condroprotectores, suplementos para ganar fuerza muscular, con el propósito de mejorar su capacidad física, de modo de posibilitar su retorno, lo que nunca ocurrió por miedo a nuevas y mas agresivas lesiones.

Un estudio reciente publicado por Filbay, Crossley \& Ackerman (2016), tuvo por objetivo evaluar e identificar los aspectos que más influenciaban la calidad de vida de los pacientes que habían sido sometidos a la reconstrucción del LCA en un periodo de 5 a 20 años de follow-up. Fueron evaluados 165 individuos con edades entre 18 a 55 años a través del cuestionario ACL-QOL. Los puntos más comúnmente identificados que influenciaban la calidad de vida, era la actividad desarrollada, las modificaciones de las actividades en el estilo de vida y el miedo a nuevas lesiones. En la evaluación de la paciente relatada en el presente estudio, los ítems que más han ejercido influencia para la baja calidad de vida detectada por la aplicación del ACL-QOL, fueron las alteraciones en el estilo de vida y principalmente el miedo a una nueva lesión; este último factor, fue decisivo para que la paciente fuese parte del $50 \%$ que desiste de su actividad deportiva, además de la presencia de lesiones asociadas instaladas en los meniscos y un cuadro de artrosis en curso. A pesar de que en el momento de la rehabilitación, los objetivos de mejoramiento de la funcionalidad, fuerza, propiocepción y entrenamiento del gesto deportivo hayan sido realizados y entrenados, la paciente continuó sin seguridad y confianza para retornar el deporte y acabó abandonandolo, detectado por el instrumento ACL-RSI. 
En relación a las evaluaciones funcionales de las rodillas, recogidas a partir de la escala de Cincinnati modificada y Lysholm, no existe un valor ideal, lo que se considera es una clasificación mínima de bueno en la suma de los puntajes finales. Los valores bajo de 30, reciben una calificación mala, entre 30 y 54, pobre, entre 55 y 79, buena y por sobre los 80 se considera excelente. $\mathrm{Al}$ analizar los valores recolectados en las evaluaciones de la paciente, se percibe que mantuvo valores considerados excelentes después del periodo de rehabilitación (2008, 2012 y 2016). Las evaluaciones con 2 , 4 y 6 meses del postoperatorio, puntuaron menos, generando clasificaciones peores, pero esas clasificaciones deben ser ponderadas por el hecho de que la paciente estuviera aún en proceso de rehabilitación e impedida de realizar determinadas actividades, que por su parte afectan la puntuación en la clasificación del cuestionario. Al analizar los valores de la escala de Lysholm, tenemos una clasificación baja para puntajes menores de 64, regular entre $65 \mathrm{y}$ 83 , buena entre 84 y 94 y excelente para valores por encima de 94 puntos. Los valores referentes a los periodos después de la rehabilitación, son todos clasificados de excelentes, corroborando la de la escala modificada de Cincinnati, así como también los valores durante el periodo de rehabilitación que se pueden justificar por el mismo motivo supracitado (Bentley et al., 2003, Peccin, Ciconelli \& Cohen, 2006).

\section{CONCLUSIONES}

Después de analizar todos los puntos arriba descritos, se percibe que las limitaciones funcionales de las rodillas de la paciente, son prácticamente inexistentes al considerar de excelentes en la escalas de evaluación utilizada, sin embargo, la misma permanece separada de las actividades de alto rendimiento e impacto. Esa afirmación puede ser mejor comprendida, después del análisis de los cuestionarios de calidad de vida y evaluación psicológica de la misma, quedando evidente la inseguridad en la realización de actividades intensas, y el miedo de una nueva reincidencia o recaída de la lesión.

Después de la realización de este estudio, queda clara la limitación, por ser un estudio de caso, pero que indica caminos claros e importantes para ser analizados y evaluados en nuevos estudios con poblaciones mayores. Es importante que la calidad de vida y la seguridad del paciente en la ejecución de actividades de trabajo durante el proceso de rehabilitación no esté limitado solo a la rehabilitación física $\mathrm{y}$ funcional.

\section{Conflicto de intereses}

Declaramos que no hubo potencial conflicto de intereses en el desarrollo de este trabajo 


\section{REFERENCIAS BIBLIOGRÁFICAS}

Almosnino, S., Brandon, SC., Day, AG., Stevenson, JM., Dvir, Z., \& Bardana, DD. (2014). Principal Component Modeling of Isokinetic Moment Curves for Discriminating between the Injured and Healthy Knees of Unilateral ACL Deficient Patients. Journal of Electromyography and Kinesiology: Official Journal of the International Society of Electrophysiological Kinesiology 24(1): 134-43. doi:10.1016/j.jelekin.2013.10.012.

Costa Astur, D., Xerez, M., Rozas, J., Vargas Debieux, P., Franciozi, C., \& Cohen, M. (2016). Anterior Cruciate Ligament and Meniscal Injuries in Sports: Incidence, Time of Practice until Injury, and Limitations Caused after Trauma. Revista Brasileira de Ortopedia: x. doi: http:// dx.doi.org/10.1016/j.rboe.2016.04.008.

Bentley, G., Biant, L.C., Carrington, R.W., et al. (2003). A Prospective, Randomised Comparison of Autologous Chondrocyte Implantation versus Mosaicplasty for Osteochondral Defects in the Knee. The Journal of Bone and Joint Surgery. British Volume 85 (2): 223-30.

Bryant, AL., Pua, YH. \& Clark, RA. (2009). Morphology of Knee Extension Torque-Time Curves Following Anterior Cruciate Ligament Injury and Reconstruction. The Journal of Bone and Joint Surgery. American Volume 91(6): 142431. doi:10.2106/JBJS.H.01335.

Danneskiold-Samsoe, B., Bartels, EM., Bulow, PM., Lund, H., Stockmarr, A., Holm, CC., Watjen, I., Appleyard, M. \& Bliddal, H. (2009). Isokinetic and Isometric Muscle Strength in a Healthy Population with Special Reference to Age and Gender. Acta Physiologica (Oxford, England) 197 Suppl: 1-68. doi:10.1111/j.1748-1716.2009.02022.x.
Filbay, SR., Ackerman, IN., Russell, TG., Macri, EM. \& Crossley, KM. (2014). Health-Related Quality of Life after Anterior Cruciate Ligament Reconstruction: A Systematic Review. Am J Sports Med 42(5): 1247-55. doi:10.1177/0363546513512774.

Filbay, SR., Crossley, KM., Ackerman, IN. (2016). Activity Preferences, Lifestyle Modifications and Re-Injury Fears Influence Longer-Term Quality of Life in People with Knee Symptoms Following Anterior Cruciate Ligament Reconstruction: A Qualitative Study. J Physiother 62(2): 103-10. doi:10.1016/j. jphys.2016.02.011.

Filbay, SR., Culvenor, AG., Ackerman, IN., Russell,TG. \& Crossley, KM. (2015). Quality of Life in Anterior Cruciate Ligament-Deficient Individuals: A Systematic Review and Meta-Analysis. British Journal of Sports Medicine 49(16): 1033-41. doi:10.1136/bjsports-2015-094864.

Garratt, AM., Brealey, S., Robling, M., Atwell, C., Russell, I., Gillespie, W., \& King, D. (2008). Development of the Knee Quality of Life (KQoL-26) 26-Item Questionnaire: Data Quality, Reliability, Validity and Responsiveness. Health and Quality of Life Outcomes 6(1): 48. doi:10.1186/1477-7525-6-48.

Gifstad, T., Sole, A, Strand, T., Uppheim, G., Grøntvedt, T., Drogset, JO. (2013). Long-Term Follow-up of Patellar Tendon Grafts or Hamstring Tendon Grafts in Endoscopic ACL Reconstructions. Knee Surgery, Sports Traumatology, Arthroscopy: Official Journal of the ESSKA 21(3): 576-83. doi:10.1007/ s00167-012-1947-0. 
Heckmann, TP., Noyes, FR., and Barber-Westin, SD. (2009). Rehabilitation of Primary and Revision Anterior Cruciate Ligament Reconstruction BT - Noyes' Knee Disorders: Surgery, Rehabilitation, Clinical Outcomes. In, Second, 303-36. Elsevier. doi:http://dx.doi. org/10.1016/B978-0-323-329033.00011-1.

Heijne, A., Hagstromer, M. and Werner, S. (2015). A Two- and Five-Year Followup of Clinical Outcome after ACL Reconstruction Using BPTB or Hamstring Tendon Grafts: A Prospective Intervention Outcome Study. Knee Surgery, Sports Traumatology, Arthroscopy: Official Journal of the ESSKA 23(3): 799-807. doi:10.1007/s00167013-2727-1.

Johnson, CC., Garcia, GH., Garner, MR. and Marx, RG. (2016). Quality of Life Following ACL Reconstruction: Baseline Predictors of Patient-Reported Outcomes. HSS Journal 12(1): 94-97. doi:10.1007/s11420-015-9473-5.

Kraus, T., Švehlík, M., Singer, G., Schalamon, J., Zwick, E., Linhart W. (2012). The Epidemiology of Knee Injuries in Children and Adolescents. Archives of $\mathrm{Or}$ thopaedic and Trauma Surgery 132 (6): 773-79. doi:10.1007/s00402-012-14800 .

Logerstedt, DS., Snyder-Mackler, L., Ritter, RC., and Axe, MJ. (2010). Knee Pain and Mobility Impairments: Meniscal and Articular Cartilage Lesions. The Journal of Orthopaedic and Sports Physical Therapy 40(6). doi:10.2519/ jospt.2010.0304.

Mohtadi, N. (1998). Development and Validation of the Quality of Life Outcome Measure (Questionnaire) for Chronic Anterior Cruciate Ligament Deficiency. The American Journal of Sports Medicine 26(3): 350-59.
Peccin, MS., Ciconelli,R., and Cohen, M. (2006). Questionário Específico Para Sintomas Do joelho 'Lysholm Knee Scoring Scale’: Tradução E Validação Para a Língua Portuguesa. Acta Ortopédica Brasileira 14: 268-72.

Rothenberg, P., Grau, L., Kaplan, L. and Baraga, MG. (2016). Knee Injuries in American Football: An Epidemiological Review. American Journal of Orthopedics (Belle Mead, N.J.) 45(6). United States: 368-73.

Shelbourne, KD., and Gray, T. (1997). Anterior Cruciate Ligament Reconstruction with Autogenous $\mathrm{Pa}$ tellar Tendon Graft Followed by Accelerated Rehabilitation: A Two- to Nine-Year Followup. The American Journal of Sports Medicine 25(6): 786 95. doi:10.1177/036354659702500610.

Swärd, P., Kostogiannis, I. and Roos, H.. (2010). Risk Factors for a Contralateral Anterior Cruciate Ligament Injury. Knee Surgery, Sports Traumatology, Arthroscopy 18(3): 277-91. doi:10.1007/ s00167-009-1026-3.

Tegner, Y., and Lysholm, J. (1985). Rating Systems in the Evaluation of Knee Ligament Injuries. Clinical Orthopaedics and Related Research, no. 198 (September): 43-49.

Trees, AH., Howe, TE., Dixon, J. \& White, L. (2011). Exercise for treating isolated anterior cruciate ligament injuries in adults. Cochrane Database of Systematic Reviews, no. 5. doi:10.1002/14651858. CD005316.pub3.

Webster, KE., Feller, JA., and Lambros, C. (2008). Development and Preliminary Validation of a Scale to Measure the Psychological Impact of Returning to Sport Following Anterior Cruciate Ligament Reconstruction Surgery. Physical Therapy in Sport: Official Journal of 
the Association of Chartered Physiotherapists in Sports Medicine 9(1): 9-15. doi:10.1016/j.ptsp.2007.09.003.

Wipfler, B., Donner, S., Zechmann, CM., Springer, J., Siebold, R., and Paessler, HH. (2011). Anterior Cruciate Ligament Reconstruction Using Patellar Tendon versus Hamstring Tendon: A Prospective Comparative Study with 9-Year Follow-Up. Arthroscopy: The Journal of Arthroscopic \& Related Surgery: Official Publication of the Arthroscopy Association of North America and the International Arthroscopy Association 27(5): 653-65. doi:10.1016/j. arthro.2011.01.015.

Wright, RW., Magnussen,RA., Dunn, WR., and Spindler, KP. (2011). Ipsilateral Graft and Contralateral ACL Rupture at Five Years or More Following ACL Reconstruction: A Systematic Review. J Bone Joint Surg Am 93(12): 1159-65. doi:10.2106/jbjs.j.00898.

Xaverova, Z., Dirnberger, J., Lehnert, M., Belka, J., Wagner, H., and Orechovska, K. (2015). Isokinetic Strength Profile of Elite Female Handball Players. Journal of Human Kinetics 49: 257-66. doi:10.1515/hukin-2015-0128.

\section{Dirección para correspondencia}

Rodrigo Okubo

Dr. Fisioterapia Ortopédica e Deportiva

Dr@ Ciencias de la Educación

Universidade do Estado de Santa Catarina, Brasil.

Contacto:

rodrigo.okubo@udesc.br 